\title{
The answer is skin deep: A case of intravascular large $B$ cell lymphoma presenting as progressive paresis and bilateral abducens nerve palsy
}

\author{
Tejas Patil ${ }^{* 1}$, Jason Mansoori ${ }^{2}$, Ryan D. Murphy ${ }^{1}$, Stephen Malkoski ${ }^{2}$ \\ ${ }^{1}$ Department of Internal Medicine, University of Colorado, Aurora, CO, USA \\ ${ }^{2}$ Department of Pulmonary and Critical Care, University of Colorado, Aurora, CO, USA
}

Received: May 31, 2016

DOI: $10.5430 /$ crcp.v3n3p72

\author{
Accepted: June 28, 2016 \\ Online Published: July 6, 2016 \\ URL: http://dx.doi.org/10.5430/crcp.v3n3p72
}

\begin{abstract}
The authors report a case of a 79-year-old, previously healthy, gentleman who presented with progressive proximal muscle weakness, petechial rash, weight loss, fatigue and diplopia. Neurologic exam demonstrated bilateral esotropia in the primary position and proximal muscle weakness in both upper and lower extremities bilaterally. Subsequent serum laboratory studies, cerebrospinal fluid analysis and neuroimaging failed to identify a unifying diagnosis. The patient was ultimately diagnosed with intravascular large B cell lymphoma (IVLBCL) via skin biopsy and subsequently underwent a single cycle of chemotherapy with rituximab, cyclophosphamide, doxorubicin, vincristine and prednisone (R-CHOP) before dying from respiratory failure. This case highlights the importance of entertaining a broad differential diagnosis for non-anatomical distribution of central nervous system (CNS) findings, especially when prior investigations into a structural cause have been unrevealing.
\end{abstract}

Key Words: Intravascular lymphoma, Cranial nerve palsy, Neuroimaging, Diplopia

\section{INTRODUCTION}

We report a case of a patient with persistent bilateral abducens nerve palsy in whom a diagnosis of intravascular large B-cell lymphoma (IVLBCL) was made via skin biopsy. IVLBCL is a rare subtype of diffuse large B-cell lymphoma (DLBCL) characterized by growth of neoplastic lymphocytes mainly within the blood vessel lumina. The clinical presentation of IVLBCL is highly variable, though cutaneous and central nervous system (CNS) manifestations are common. ${ }^{[1]}$ Neurological symptoms noted in patients with IVLBCL are heterogeneous, and alteration of consciousness, motor and sensory deficits, seizure, paresis, dementia, intentional tremor, disorientation, and gait disturbance have been described. ${ }^{[1,2]}$ Cranial nerve palsies are an exceedingly rare presentation of IVLBCL. ${ }^{[3,4]}$ This case illustrates an uncommon presentation of a rare disease and reinforces the importance of sustaining a broad differential diagnosis while evaluating non-anatomic distribution of CNS findings.

\section{Case presentation}

A 79-year-old gentleman with no significant past medical history presented with five months of progressive global weakness, weight loss and fatigue. His weakness initially involved the proximal upper muscles, though lower extremity involvement subsequently ensued. Two months prior to admission, the patient developed sudden onset of diplopia.

\footnotetext{
*Correspondence: Tejas Patil, MD; Email: tejas.patil@ucdenver.edu; Address: University of Colorado School of Medicine, 12631 East 17th Avenue, B177, Aurora, CO 80045, USA.
} 
In addition, the patient's wife noted a faint periumbilical rash. He denied constitutional symptoms, headaches, vertigo, slurred speech, photophobia, phonophobia, facial pain, nausea, orthopnea, paroxysmal nocturnal dyspnea, anorexia, arthralgias, polyuria or polydipsia.

The patient went to his primary care physician for evaluation of diplopia. During this visit, he was noted to have an erythrocyte sedimentation rate $(\mathrm{ESR})$ of $81 \mathrm{~mm} /$ hour and a $\mathrm{C}$ reactive protein (CRP) of $18.1 \mathrm{mg} / \mathrm{dl}$. He was empirically started on high dose oral prednisone given suspected giant cell arteritis. This resulted in temporary improvement of the patient's symptoms, though he continued to need a cane for ambulation. Unfortunately, his progressive muscle weakness and diplopia returned after several weeks of oral prednisone therapy.

He had no recent or past exposure to cigarette smoke. He had no prior contact with individuals known to have tuberculosis. He reported no recent international travel. He reported no prior occupational exposure to asbestos, silica, beryllium or other industrial chemicals. He had no history of diabetes, peripheral vascular disease, hypertension or coronary artery disease. He had no prior history of connective tissue disease, congenital immune deficiency or history of organ transplant. He maintained an active lifestyle and skied multiple times a week prior to onset of symptoms. Past surgical, social and family histories were non-contributory.

The patient was admitted to our institution for evaluation of his persistent weakness and diplopia. On examination, he appeared tired, but in no apparent distress. His temperature was $36.3^{\circ} \mathrm{C}$, pulse was 97 times/min, respiratory rate was 18 times/min, blood pressure was $113 / 61 \mathrm{mmHg}$, and his oxygen saturation was $96 \%$ on $4 \mathrm{~L}$ via nasal cannula. There was no evidence of pallor, lymphadenopathy, clubbing, joint swelling or edema. Aside from sinus tachycardia, cardiopulmonary exam was unremarkable. Abdominal exam did not reveal hepatosplenomegaly. There was a purpuric rash along the left abdomen with well-demarcated borders and scattered petechiae (see Figure 1).

His ocular exam was notable for horizontal diplopia without loss of visual fields. Pupils were round, equal and reactive to light without any aniscoria. There was no ptosis or abnormalities of the eyelid. Funduscopic exam, as performed by a neuro-ophthalmologist, was unremarkable. The patient had esotropia in the primary position with an inability to abduct both eyes, consistent with bilateral abducens (VI) nerve palsy. There was no slowing of the adducting saccades and no nystagmus of the abducting eye to suggest internuclear ophthalmoplegia. Aside from the abducens nerve palsy, the remainder of the cranial nerve exam was normal. Muscle tone was normal without any fasiculations, resting tremor, or spasticity. Strength was 5/5 distally, but 4/5 in the proximal muscles of both upper and lower extremities bilaterally. Deep tendon reflexes were $2+$ and symmetric. There was no clonus or asterixis. There was no pronator drift.

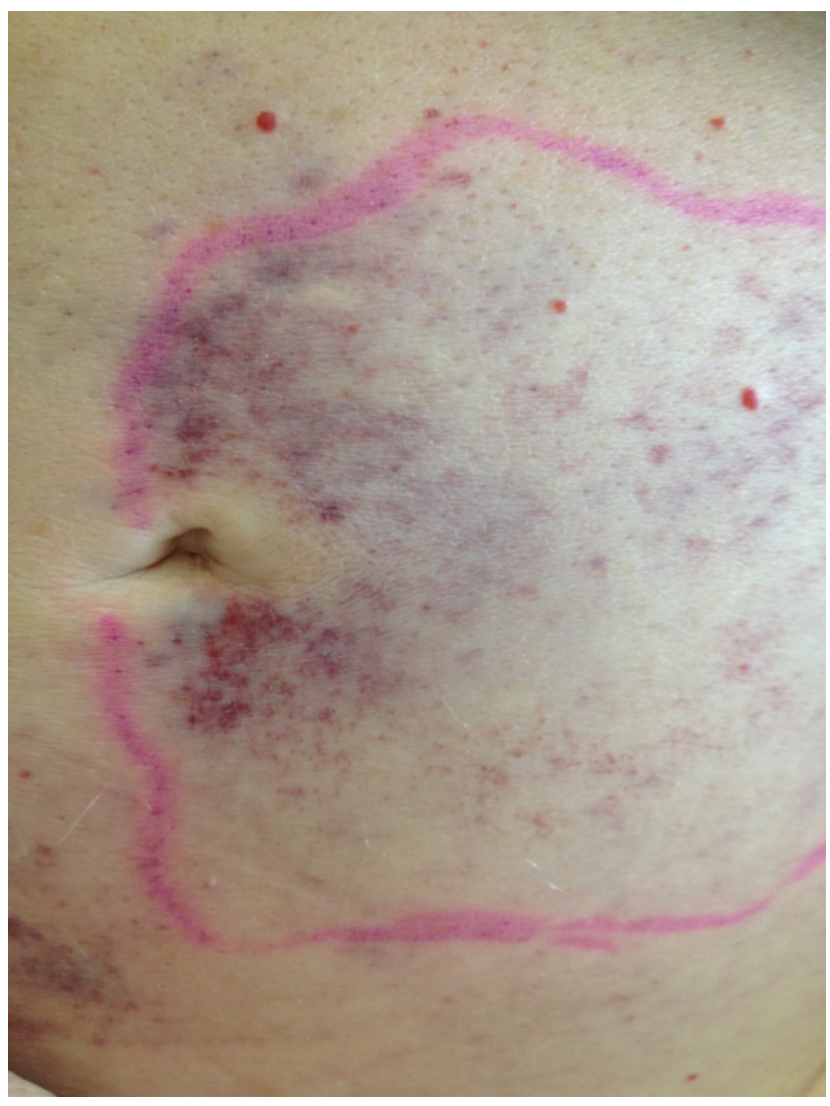

Figure 1. A purpuric rash along the left abdomen with well-demarcated borders and scattered petechiae

Admission laboratory studies were notable for hyponatremia $\left(\mathrm{Na}^{+} 126 \mathrm{mmol} / \mathrm{L}\right)$ and normocytic anemia (Hgb $\left.9.2 \mathrm{~g} / \mathrm{dl}\right)$ without additional cytopenias. Liver function tests, coagulation studies, thyroid function tests, hemoglobin A1C, lipid panel, creatinine kinase and aldolase were within normal limits. Chest radiography was normal. Admission blood and respiratory cultures demonstrated no growth after several days. HIV and HCV antibodies were negative.

An elevated ESR (81 mm/hour) and CRP (18 mg/dl) on admission prompted evaluation for vasculitis; however antinuclear antibody, ANA, rheumatoid factor, anti-DNA, antiSmith, anti-RNP, anti-SSA/SSB, anti-centromere, c-ANCA, p-ANCA, C3 and C4 were all within normal limits. A serum electrophoresis demonstrated a non-specific lambda light chain band, but the $\kappa: \lambda$ ratio was normal. A skeletal survey did not show lytic lesions and bone marrow biopsy demonstrated trilineage hematopoesis with no abnormal populations 
by flow cytometry. Electromyogram and nerve conduction velocity (EMG/NCV) testing revealed only a mild sensory axonal polyneuropathy that could not account for the patient's proximal motor weakness.

A lumbar puncture was performed and the cerebrospinal fluid was notable for glucose of $101 \mathrm{mg} / \mathrm{dl}$, total protein $103 \mathrm{mg} / \mathrm{dl}, 2 \mathrm{WBC} \mathrm{m^{3 }}, 2 \mathrm{RBC} \mathrm{m^{3 }}$ and an opening pressure of $64 \mathrm{mmH}_{2} \mathrm{O}$. Viral serologies, oligoclonal banding, and paraneoplastic panel were negative. Cytology and flow cytometry did not demonstrate evidence of hematologic malignancy in the cerebrospinal fluid.

Computed tomography (CT) of his chest, abdomen and pelvis did not demonstrate any evidence of masses, organomegaly, or lymphadenopathy. A brain magnetic resonance imaging (MRI) with gadolinium contrast demonstrated mild cerebral volume loss with scattered white matter disease in the supratentorial brain and pons that was nonspecific but presumably related to chronic microvascular ischemia. There were no signal abnormalities to suggest limbic encephalitis, leptomeningeal disease or paraneoplastic syndromes. Colonoscopy and esophagogastroduodenoscopy were performed prior to admission to evaluate the patient's weight loss and these studies did not reveal any masses or strictures.

Given the absence of a unifying diagnosis, the patient underwent a skin biopsy of the abdominal rash shown in Figure 1. Atypical and enlarged cells were seen occluding the small vessels of the dermis and subcutis. Immunohistochemistry identified these cells as CD20 positive, consistent with a diagnosis of IVLBCL (see Figures 2-3).

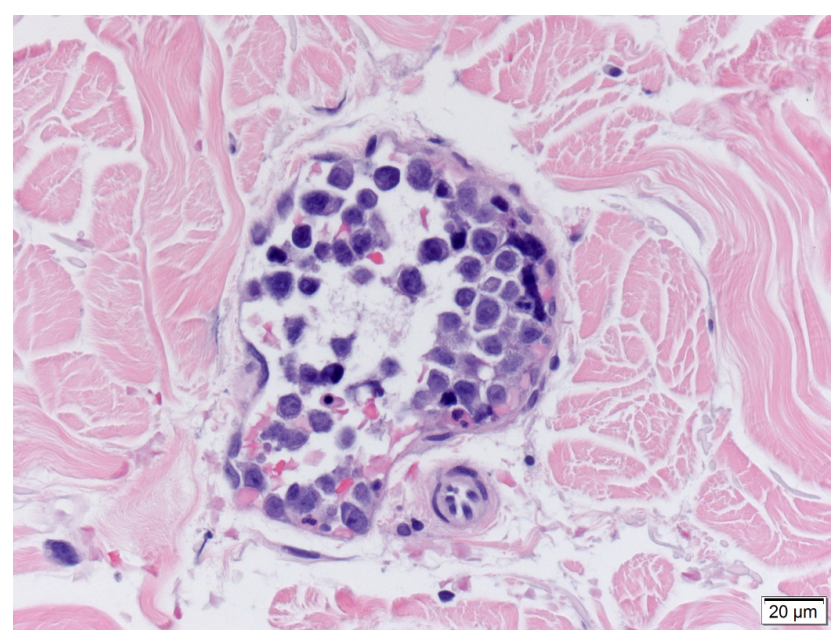

Figure 2. Atypical and enlarged cells occlude small vessels with the dermis and subcutis (left, Hematoxylin and Eosin)

The patient received chemotherapy with rituximab (375 $\mathrm{mg} / \mathrm{m}^{2} /$ day at $50 \mathrm{mg} /$ hour), cyclophosphamide
(750 mg/m²/day), doxorubicin $\left(50 \mathrm{mg} / \mathrm{m}^{2} /\right.$ day $)$, vincristine ( $2 \mathrm{mg} /$ day), and oral prednisone $(100 \mathrm{mg} /$ day). Unfortunately, the patient developed severe hypotension and hypoxia with his first rituximab infusion. He subsequently needed intubation with mechanical ventilation. After discussion with the patient's family, we elected for dose-reduced CHOP therapy without rituximab. The patient's hypoxia improved with dose-reduced CHOP therapy over the course of one week and he was subsequently extubated after completing a full cycle. Despite this intervention, the patient continued to have progressive respiratory failure and died in the ensuing days.

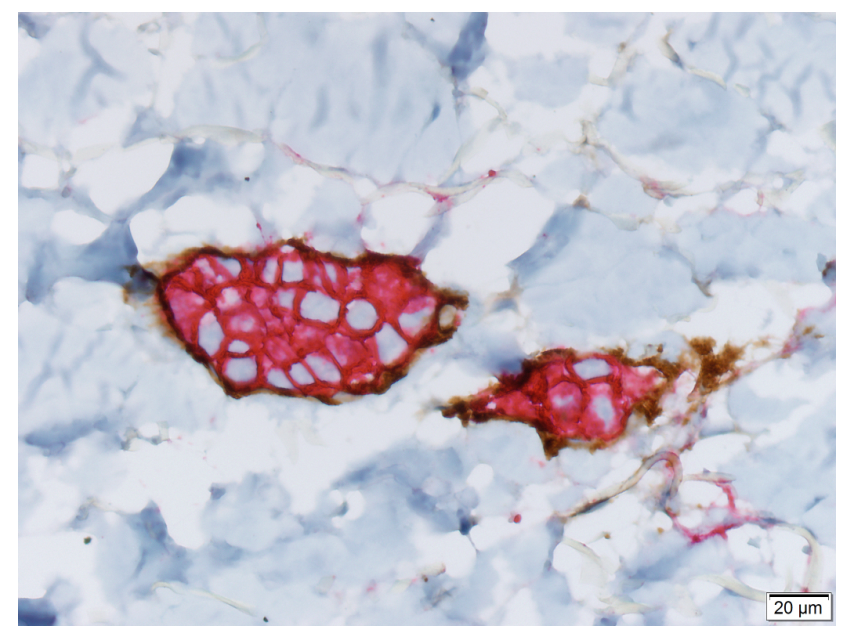

Figure 3. A dual immunohistochemical study for endothelial cells in brown (CD31) and labels the lymphomatous cells with a red chromagen (CD20) (right)

\section{Discussion}

Although IVLBCL frequently involves both the CNS and peripheral nerve microvasculature, the neurological presentation of IVLBCL can be highly variable. ${ }^{[1,2]}$ The CNS manifestations tend to be subacute and progressive. They include, in order of frequency: 1) subacute encephalopathy 2) disorders of the lumbosacral cord, 3) cerebral infarcts or stroke-like symptoms and 4) seizures. ${ }^{[1]}$ The specificity of clinical neurological testing remains low. ${ }^{[2]}$ Lumbar puncture rarely establishes the diagnosis. ${ }^{[1,2,5]}$ Neuroimaging findings are non-specific and imaging abnormalities are detected in about half of patient with IVLBCL who present with neurologic symptoms. ${ }^{[5,6]} \mathrm{CT}$ is generally non-diagnostic, though MRI can show non-specific hyperintensity of white-matter lesions suggestive of small-vessel ischemic disease or demyelination. ${ }^{[1,5,6]}$

Given that IVLBCL typically presents as a disseminated disease, no standard procedure for organ biopsy has been established for this disease. ${ }^{[5]}$ In one series, bone marrow 
involvement was observed in one third of patients with IVLBCL. ${ }^{[7]}$ Intravascular lymphoma cells typically demonstrate a 'mature' (i.e., not precursor) lymphocyte immunophenotype characteristic, with over $90 \%$ demonstrating B-cell phenotype with CD20 expression. ${ }^{[8]}$ Cytogenetic analysis in the literature is very limited, but in a single case series, the most frequent abnormality was the accumulation of structural aberrations in chromosomes 1,6 , and 18 , especially $1 \mathrm{p}$ and trisomy $18 .{ }^{[9]}$ The most distinctive characteristic regarding IVLBCL is their tendency to grow within blood vessel lumina without any substantive extravastation. These cells seem to lack critical molecules, such as CD29 ( $\beta 1$ integrin subunits), CD54 (intercellular adhesion molecule) and CD11a that are vital for extravastation. ${ }^{[8]}$ The prognostic role of bone marrow histology, immunophenotype characteristics and cytogenetics for IVLBCL has yet to be clearly defined.

Given the rarity of the disease, there are no randomized control trials comparing treatments for IVLBCL. Objective responses after cyclophosphamide, doxorubicin, vincristine, and prednisone (CHOP) or CHOP-like chemotherapy have been reported. ${ }^{[10,11]}$ Anthracycline-based chemotherapy is associated with nearly a $60 \%$ response rate and a 3 -year overall survival rate higher than $30 \% .{ }^{[10-12]}$ Ferreri and coworkers reported clinical outcomes for 22 patients who received chemotherapy with 3-year overall survival of 33\% ${ }^{[12]}$ Shimada and colleagues conducted a retrospective review of 57 patients who received chemotherapy with and without rituximab. The progression-free survival and overall survival at 2 years for patients receiving rituximab with chemotherapy was $56 \%$ and $66 \%$ respectively. In patients who received chemotherapy without rituximab, progression free survival and overall survival at 2 years were $27 \%$ and $46 \%$ respectively. ${ }^{[11]}$ There are case reports that using more intensive chemotherapy regimens such as m-BACOD (methotrexate, bleomycin, doxorubicin, cyclophosphamide, vincristine, and dexamethasone) or autologous stem cell transplantation may improve long-term survival. ${ }^{[13]}$ The combination of anthracycline-based chemotherapy and rituximab may be helpful in prolonging overall survival, though the prognosis for IVLBCL continues to remain poor despite these interventions. It is unclear whether the poor prognosis is because IVLBCL is biologically more aggressive than other forms of diffuse large B cell lymphomas or because this disease is frequently diagnosed at a very advanced stage.

Toxic effects associated with administration of rituximab should be mentioned. The characteristic of tumor-cell growth in the lumina of small vessels can lead to severe immunoreactions with rituximab infusion. In fact, severe pulmonary complications related to rituximab infusion as an initial treat- ment have been reported. ${ }^{[11,14,15]}$ The pathophysiology of this reaction has been attributed to the release of cytokines secondary to cross-linking and release of target cell cytokines (such as IFN- $\gamma$, IL-6, IL-8, and TNF- $\alpha$ ) within two hours of infusion. ${ }^{[15]}$ Makino and colleagues performed a review of the literature describing all cases of rituximab-infusion reactions from 1994-2011 and observed that patients who received rituximab during the first course of therapy developed significantly more adverse effects, with five out of fourteen patients developing hypoxic respiratory failure. ${ }^{[15]}$ This study did not specifically identify whether the patients who developed adverse effects had more comorbidities and multi-organ involvement prior to initiation of rituximab.

The salient symptoms in this case include progressive proximal muscle weakness, weight loss, and new onset of diplopia in an elderly, though otherwise healthy, gentleman without any clear occupational or environmental risk factors. His neurological exam demonstrated bilateral esotropia in the primary position and bilateral proximal muscle weakness. In elderly patients, isolated abducens nerve involvement is most often idiopathic, though when a cause is ascertained, it is usually secondary to small vessel involvement such as vascular disease or diabetes. Neuroimaging should be obtained to exclude neoplasms involving the base of the skull, such as nasopharyngeal carcinoma, pituitary adenomas, cavernous sinus meningioma, or metastatic encroachment of the abducens nerve. Finally, abducens nerve palsy can be a rare manifestation of systemic processes such as multiple sclerosis, tertiary syphilis, systemic lupus erythematosus, sarcoidosis and hematological malignancies. This case highlights the challenges associated with correctly diagnosing IVLBCL. It is important to consider a broad differential diagnosis in the evaluation of non-anatomic distribution of CNS findings, especially when prior investigations fail to reveal an obvious structural cause.

\section{ACKNOWLEDGEMENTS}

All authors contributed towards the article by making substantial contributions to conception, design, acquisition of data, or analysis and interpretation of data. Tejas Patil was involved in the conception, design, and writing of the manuscript. Tejas Patil, Jason Mansoori, and Stephen Malkoski participated in collection of clinical data and the initial draft of the manuscript. Ryan D. Murphy was involved in the initial work-up of this patient and assisted with revision of the manuscripts.

\section{CONFlicts of InTEREST Disclosure}

The authors declare no competing interests. 


\section{REFERENCES}

[1] Beristein X, Azzarelli B. The neurological masquerade of intravascular lymphomatosis. Arch Neurology. 2010; 59(3): 439-43. http: //dx.doi.org/10.1001/archneur.59.3.439

[2] Glass J, Hochberg FH, Miller DC. Intravascular lymphomatosis a systemic disease with neurologic manifestations. Cancer. 1993; 71: 3156-64. http://dx.doi.org/10.1002/1097-0142(1993 0515) $71: 10<3156:$ : AID-CNCR2820711043>3.0. CO ; $2-0$

[3] Torrenbeck R, Scheltens P, Strack van Schijindel RJ, et al. Angiotropic intravascular large-cell lymphoma with massive cerebral extension. J Neurol Neurosurg Psychiatry. 1993; 56: 914-6.

[4] Sukipanichnant S, Visuthisakchai S. Intravascular lymphomatosis: a study of 20 cases in Thailand and review of literature. Clin Lymphoma Myeloma. 2006; 6(4): 319-28. PMid: 16507210. http: //dx.doi.org/10.3816/CLM. 2006.n.007

[5] Shimada K, Kinoshita T, Naoe T, et al. Presentation and management of intravascular large B-cell lymphoma. Lancet Oncol. 2009; 10: 895902. http://dx.doi.org/10.1016/S1470-2045 (09)70140-8

[6] Baehring JM, Henchcliffe C, Ledezema CJ, et al. Intravascular lymphoma: magnetic resonance imaging correlates of disease dynamics within the central nervous system. J Neurol Neurosurg Psychiatry. 2005; 76: 540-4. PMid: 15774442. http://dx.doi.org/10.11 36/jnnp. 2003.033662

[7] Ferreri AJM, Elías C, John FS, et al. Intravascular lymphoma: clinical presentation, natural history, management and prognostic factors in a series of 38 cases, with special emphasis on the 'cutaneous' variant. Brit J Haematol. 2004; 127(2): 173-83. PMid: 15461623. http://dx.doi.org/10.1111/j.1365-2141.2004.05177.x

[8] Ponzoni M, Ferreri AJM. Intravascular lymphoma: a neoplasm of the 'homeless' lymphocytes? Hematol Oncol. 2006; 24(3): 105-12. PMid: 16721900. http://dx.doi.org/10.1002/hon.776
[9] Tsukadaira A, Okubo Y, Ogasawara H, et al. Chromosomal aberrations in intravascular lymphomatosis. Am J Clin Oncol. 2002; 25(2): 178-81. PMid: 11943898. http://dx.doi.org/10.1097/00000 421-200204000-00015

[10] Ponzoni M, Andrés JMF, Elías C, et al. Definition, diagnosis, and management of intravascular large B-cell lymphoma: proposals and perspectives from an international consensus meeting. J Clin Oncol. 2007; 25(21): 3168-73. PMid: 17577023. http://dx.doi.org/1 $0.1200 /$ JCO. 2006.08 .2313

[11] Shimada K, Matsue K, Yamamoto K, et al. Retrospective analysis of intravascular large B cell lymphoma treated with rituximabcontaining chemotherapy as reported by IVL study group in Japan. $\mathbf{J}$ Clin Oncol. 2008; 26: 3189-95. PMid: 18506023. http://dx.doi .org/10.1200/JC0.2007.15.4278

[12] Ferreri AJM, Dognini GP, Bairey O, et al. The addition of rituximab to anthracycline-based chemotherapy significantly improves outcome in "Western" patients with intravascular large B-cell lymphoma. Brit J Haematol. 2008; 143(2): 253-7. PMid: 18699850. http://dx.doi.org/10.1111/j.1365-2141.2008.07338.x

[13] Zuckerman D, Selium R, Hochberg E. Intravascular lymphoma: The Oncologist's "Great imitator." The Oncologist. 2006; 11(5): 496-502. PMid: 16720850. http://dx.doi.org/10.1634/theoncologi st. 11-5-496

[14] Wu SJ, Chou WC, Bo-Sheng K, et al. Severe pulmonary complications after initial treatment with rituximab for Asian-variant of intravascular lymphoma. Haematologica. 2007; 92: 141-2. PMid: 17229654. http://dx.doi.org/10.3324/haematol.10561

[15] Makino K, Nakata J, Kawachi S, et al. Treatment strategy for reducing risk of rituximab-induced cytokine release syndrome in patients with intravascular large B cell lymphoma: a case report and review of the literature. Journal of Medical Case Reports. 2013; 7: 280. PMid: 24377366. http://dx.doi.org/10.1186/1752-1947-7-280 